\title{
Establishing cocooning apple leafcurling midge onto apples for disinfestation research
}

\author{
A. J. Hawthorne, M.J. Griffin, N.E.M. Page-Weir, S.P. Redpath and L.E. Jamieson \\ Plant \& Food Research, Private Bag 92169 Auckland 1142 New Zealand \\ Corresponding author: amanda.hawthorne@plantandfood.co.nz
}

Apple exports intercepted with cocooning apple leafcurling midge (ALCM) are fumigated with methyl bromide (MB), which reduces apple quality. ALCM is controlled in the orchard using the insecticide Movento $^{\mathrm{Tm}}$, while also being parasitised by Platygaster demades and preyed on by generalist predators such as Sejanus albisignata. These ALCM control measures make it difficult to collect sufficient numbers of ALCM-infested apples for disinfestation research on alternatives to MB. Therefore, procedures were developed for collecting and storing cocooning ALCM on apples in the laboratory. ALCM-infested leaves were collected in plastic bags, saturated with water and placed at $20^{\circ} \mathrm{C}$. The water stimulated larvae to exit the leaf-rolls. Using a fine paint brush, ten larvae were placed on the calyx of an apple and confined using a cage attached with Blu-Tak'. The apples were placed in plastic bins and sprayed with water. A mesh net and a brown paper bag were secured over the top of the bin to reduce condensation. The bin was stored at $20^{\circ} \mathrm{C}, 16: 8$ light:dark for 10-15 days. Of 1,133 apples established, 988 of them (87.2\%) had on average 2.2 cocooning ACLM. Large numbers of cocooning ALCM on fruit can now be provided for disinfestation research.

\section{A simple method for conidial production and for inoculating apples with Neofabraea alba}

\author{
I.P.S. Pushparajah, B.M. Fisher, P.N. Wood and K.R. Everett \\ Plant \& Food Research, Private Bag 92169 Auckland 1142 New Zealand \\ Corresponding author: kerry.everett@plantandfood.co.nz
}

Current methods for producing conidia of Neofabraea alba, the fungal cause of Bull's eye rot of apple, are laborious and time-consuming. A minimum of 6 weeks is required. Mycelial cultures of N. alba did not produce conidia on commonly used potato dextrose agar, and therefore several other media were tested. Growth on corn meal agar resulted in a high conidial yield (ca $10^{6}$ conidia/ml) after 5-7 days growth. Published research reports a method to inoculate apples with this pathogen without wounding, but it requires specialised equipment. A simple method that does not require specialised equipment was developed to inoculate apple fruit with the Bull's eye rot pathogen without wounding. Conidia were placed on water agar, and apple fruit were placed on these spores for at least 5 days for establishment of infections. Reliable infection of $70-80 \%$ of apples required a 14 -day wetness period after the fruit were placed on the conidial solution. This wound-free N. alba inoculation method provides the basis for further research to examine the effect of various treatments designed to reduce the impact of this pathogen in apple orchards. 